\title{
Paravertebral Abscess Caused by Bukholderia Pseudomallei in
}

\author{
S Ahmad, MBBS, L Azura, MS Ortho, S Duski, MS Ortho, MY Aziz, MS Ortho \\ Department of Orthopaedics, Sultan Haji Ahmad Shah Hospital, Temerloh, Malaysia
}

\begin{abstract}
A 53-year-old Malay man was admitted with intestinal obstruction, fever and lower limb weakness. Initial clinical impression was myelitis causing paralytic ilues and paraperesis. Blood culture showed Burkholderia pseudomallei infection and subsequent MRI showed paravertebral abscess. This case highlights a rare manifestation of melioidosis involving the spine and difficulties in establishing the diagnosis.
\end{abstract}

\section{INTRODUCTION}

Melioidosis is an infectious disease is caused by the gramnegative saprophyte, Burkholderia pseudomallei. This pathogen is commonly found in soil and water in Asia Pacific region, and the highest rates of diseases currently are reported in Thailand and Australia ${ }^{1}$. The incidence of Meliodosis in Malaysia as a whole is not known, but the reported rate for the state of Pahang is 6.1 per 100,000 population per year ${ }^{1}$.

Spinal involvement is rare in melioidosis. In fact, of the 50 cases of melioidosis at the University Malaya Medical Centre, Kuala Lumpur over the past 30 years, only three involved the central nervous system ${ }^{2}$. This report highlights a case of melioidosis with spinal involvement that occurred.

\section{CASE STUDY}

A 53-year-old mechanic with a history of diabetes mellitus was admitted complaining of backache over the last 2 months. Two weeks prior to admission, he developed high grade fever and bilateral lower limb weakness and poor bowel and urinary control. He was admitted for progressive abdominal distension with intermittent pain

On admission, his temperature was 38.5 ; he was alert but appeared septic. His blood pressure and pulse rate were within normal range. The abdominal distension was suggestive of intestinal obstruction. There was no tenderness over the back or any evidence of paravertebral spasm. Neurological status, according to the American Spinal Cord Injury Association (ASIA) standard neurological classification $^{3}$ patchy. It was zero for both the lower limbs and reduced sensation was one at the level of lumbar vertebra one through eight Lower limbs were hyper-reflexive with presence of ankle clonus. Peri-rectal examination showed reduced superficial and deep anal sensation with lax anal tone. Blood work showed normal white blood cells count at $6.4 \times 1000 /$ LL with neutrophils predominant. Erythrocytes sedimentation rate (ESR) was $119 \mathrm{~mm} / \mathrm{hr}$., alkaline phosphatase was significantly elevated (236; normal value : 20-90). All others blood tests were normal. Initial abdominal radiograph showed evidence of ileus with degenerative changes of the spine. The patient was started was started empirically on Imipenem and Metronidazole and treated as intestinal obstruction with myelitis.

Day 4 after admission and resting of the bowel, repeat abdominal radiograph revealed soft tissue swelling of the lower thoracic spinal region (Fig.1). Urgent MRI (Fig 2) revealed paravertebral abscess at the level of T8 and T9 thoracic vertebra with suspicion of epidural extension. The patient went into shock shortly after the MRI procedure and required inotropic support. At this time blood cultures revealed Burkholderia pseudomallei with sensitivity to Trimethoprim, Ceftazidime, Cefotaxime and AmoxiClavulanate acid. Antibiotics were changed to Ceftazidime and Amoxi-Clavulanate. Mantoux test was negative.

The condition eventually stabilized and patient underwent spinal decompression on day 7 of admission. Surgical findings indicated a swollen spinal cord, thickened ligamentum flavum and pus at the base of the T9 and T10 vertebra. Intra-operative tissue culture showed no growth. There was minimal improvement of the neurological status after surgery and the patient was eventually discharged after completion of one month of intravenous antibiotics. He was put on additional five months of oral antibiotics at home.

On follow up, there was slight improvement of his neurological status. At a six month follow-up appointment, his neurological status according to ASIA charting was improved: right sided sensation improved from one to two at the level of the eighth thoracic vertebra, motor function on the right side also improved from grade zero to grade two at the level of the second lumbar vertebra. However, the deficit over the left lower limb remained. He has no bowel or urinary incontinence at follow-up and wheelchair bound. 


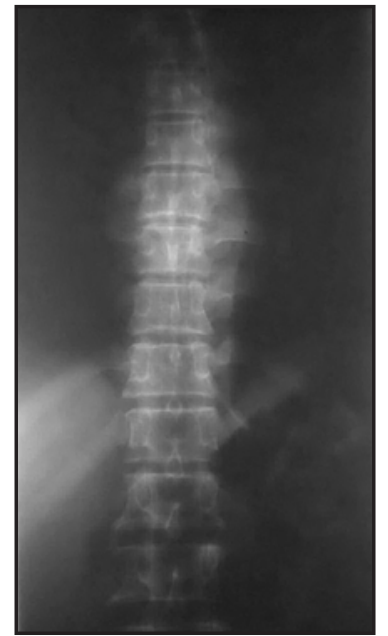

Fig. 1: Plain thoracolumbar radiograph showing paravertebral soft tissue swelling.

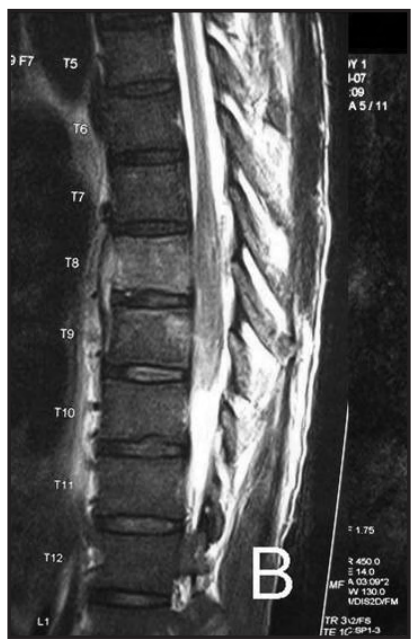

Fig. 2B: T2-Weighted MRI film showing hyperintense lesion involving the body and pedicle of eight and nine thoraxic vertebra.

\section{DISCUSSION}

This case report highlights a spinal infection at the level of thoracic region caused by Burkholderia pseudomallei. Although the infection at any site of the body is possible, spinal involvement is a rare. In the 50 case series of melioidosis reported for University Malaya Medical Centre, Kuala Lumpur over 30 years, only 3 cases involved the central nervous system and of those, only one with a spinal abscess $^{2}$. Of 14 cases of spinal pyogenic infection reported by Nather at al, only one was caused by Burkholderia pseudomallei ${ }^{4}$. Melioidosis is more commonly seen in people who have predisposing factors such as diabetes1, excessive alcohol intake (39\%), chronic lung disease (27\%) and chronic renal disease, and all these co morbid factors are associated with an increase risk of mortality ${ }^{1}$.

In this case, there is a delay in the diagnosis of spinal pathology due to coexisting abdominal problem and general sepsis. Neurological deficit of the lower limbs was initially

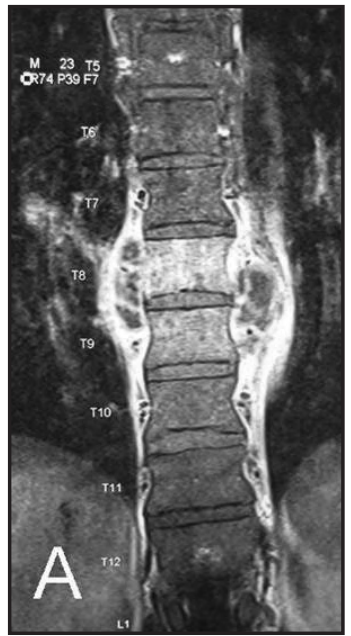

Fig. 2A:MRI film of lower thoracic spine showing of paravertebral soft tissue swelling with hyperintense signal.

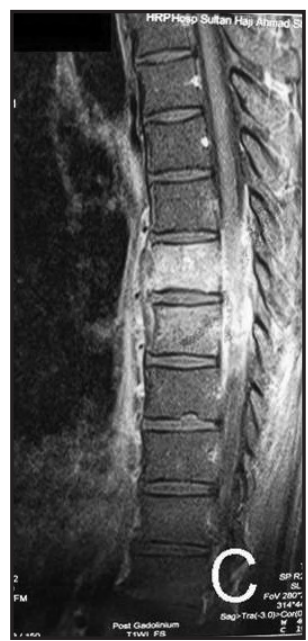

Fig. 2C: Post gadolinum MRI film showing enhancement of the spinal abscess.

attributed to myelitis because of the pathy nature of motor and sensory deficit. An early surgical decompression and an extended course of combination antibiotic therapy would have been the optimal treatment for this patient ${ }^{4}$. Failure to complete treatment or treatment with shorter course of antibiotics ( $<8$ weeks) may results in relapse of infection. Combination antibiotics treatments have been shown to produce better outcome and lower relapse rates ${ }^{5}$. The usage of Ceftazidime during the initial phase has been shown to reduce the mortality rate to almost half 5 .

Generally, patients with melioidosis involving the central nervous system may have a good outcome if there is early diagnosis, early treatment, appropriate surgical intervention and completion of 6 months of antibiotics therapy. High level of suspicion, repeated evaluation and investigation are all essential components of management that may have improve the outcome of this condition. 


\section{REFERENCES}

1. How SH, Liam CK. Melioidosis. A Potentially Life Threatening Infection. Med J Malaysia 2006; 61(3): 386-95.

2. Muthusamy KA, Waran V, Puthucheary SD. Spectra of central nervous system melioidosis. J Clin Neurosci. 2007; 14(12): 1213-5.

3. Maynard FM Jr, Bracken MB, Creasey G, Ditunno JF, Donvan WH, Ducker TB et al. International Standards for neurological and Functional Classification of spinal court injury. Spinal Cord 1997; 35:266-274.

4. Nather A, David V, Hee HT, Thambiah J. Pyogenic vertebral osteomyelitis: a review of 14 cases. J Orthop Surg 2005; 13(3):240-4.

5. Chaowagul W. Relapse in melioidosis: incidence and risk factors. J Infect Dis 1993; 168(5): 1181-5. 